\title{
TESIS DOCTORALES
}

$\square \square \square$ 
CARRASCOSA CEBOLLA, María Jesús: Prevención de las Conductas Disruptivas en el Alumnado y Descenso del Índice de Ansiedad y Estrés del Profesor. Departamento de Teoría de la Educación. Universidad de Valencia. Abril de 1996. (Tesis dirigida por el Dr. D. Bernardo Martínez Mut, presidida por el Dr. D. Juan Escámez Sánchez.)

El objetivo primordial de esta tesis es diseñar, llevar a cabo y evaluar un programa de intervención educativa que prevenga las conductas disruptivas en el alumnado, forme a los padres en estilos de supervisión y de apoyo al profesorado, con pautas y métodos operativizados que repercutan en su autoconcepto personal y profesional.

El trabajo se estructura en seis capítulos. En los capítulos I y II, se fundamenta el marco teórico-conceptual que da el soporte a la investigación. Se realiza un análisis de las técnicas metodológicas de gestión empresarial que posibilitan la implicación del personal y la mejora de la calidad del trabajo que se realiza habitualmente, como son los programas de participación del personal, o los Círculos de Calidad, y se aplican por primera vez al Sistema Educativo.

Se acota el objeto de estudio "la indisciplina", y se describe su evolución a lo largo de la historia, su etiología y principales pautas de tratamiento.

El capítulo tercero relata: a) El diseño del plan de investigación; b) Los instrumentos de medida (en el ámbito de los alumnos: IPE, Inventario de Problemas Escolares, Cuestionario de opiniones y expectativas de los alumnos, y Cuestionario de Conductas Disruptivas; en cuanto al ámbito de los profesores: ISRA, Inventario de Situaciones y Respuestas de Ansiedad, y EAE Escala de Apreciación del Estrés; y en cuanto al ámbito de las familias: Cuestionario de Rasgos Familiares, y Cuestionario de Estilo de Supervisión de los padres); c) La muestra de nuestro estudio es el segundo Ciclo de Educación Primaria; d) Se enuncian las hipótesis de trabajo; e) Las metas a conseguir y $f$ ) Se describen los criterios de evaluación.

El capítulo cuarto se dedica al análisis de la realidad previa a la ejecución del programa.

En el capítulo quinto se describe la planificación y ejecución del programa. La metodología utilizada se basa en la reflexión y autocontrol que posibilita que el alumno aprenda a analizar las consecuencias de sus acciones y decida qué tipo de conductas o variables comportamentales desea eliminar, potenciar o desarrollar según sus intereses personales, escolares $\mathrm{y} / \mathrm{o}$ familiares.

Se realizan veinte sesiones de trabajo con los alumnos, distribuidas en cuatro bloques. El primer bloque está destinado a corregir comportamientos. El segundo, a que el alumnado tome conciencia de su realidad. El bloque tercero, al desarrollo del autocontrol, y el cuarto, a que los alumnos vivencien y aprendan a controlar las consecuencias de sus acciones por medio del juego y la dramatización.

Cada sesión de trabajo viene operativizada en forma de fichas donde aparece: el objetivo, las actividades y el material a utilizar.

El capítulo sexto, detalla los resultados obtenidos tras la ejecución del programa. Éstos confirman las hipótesis planteadas, y encontramos que los alumnos que han seguido el programa han superado problemas conductuales en senti- 
do amplio como son agresividad, hiperactividad, desobediencia y oposicionismo, que alteran la dinámica de la clase como los comportamientos inatentivos que dispersan la atención y el interés por las actividades curriculares.

Por otro lado, manifiestan una actitud más positiva hacia el sistema escolar.

Los alumnos se muestran menos retraídos, no se detectan conductas de aislamiento o inhibición social. Por lo tanto, han desarrollado habilidades sociales.

$Y$ aunque nuestro objetivo no era mejorar los problemas de aprendizaje, vemos como éstos han descendido y la adquisición de conocimientos es más fluida.

Detectamos cómo los alumnos agradecen, desean y valoran las normas de convivencia, y en la relación con sus compañeros y en el clima del aula, priorizan el respeto, el orden y el trabajo. A la par que piden al tutor coherencia en sus funciones y en su actitud personal ("que no cambie de humor" y "que sea justo al poner las notas").

Los profesores, se han implicado en la ejecución del programa, y hemos detectado un descenso del índice de ansiedad y estrés en el desempeño de sus actividades laborales.

$Y$ en cuanto al ámbito de las familias, hemos conseguido que los padres adquieran criterios de supervisión. El $80 \%$ afirma que ha modificado su forma de actuar.

CASARES GARCÍA, Pilar M.: Los valores del profesorado en formación y su incidencia educativa. Departamento de Pedagogía. Universidad de Granada. Diciembre de 1998 (Tesis Doctoral dirigida por los doctores D. Enrique Gervilla Castillo y $\mathrm{D}^{\mathrm{a}}$ Leonor
Buendía Eisman. Presidida por el Dr. D. Paciano Fermoso Estébanez.)

Esta tesis está motivada por un interés y un convencimiento personales acerca de los valores y, más concretamente, acerca de la educación como vía de transmisión de valores. De acuerdo con el marco legal, nuestro sistema educativo formal pretende trasmitir un conjunto de valores que responda a un modelo integral de persona. Uno y otro aspecto: educación de valores y educación integral, se consideran criterios de calidad de la educación.

En una sociedad como la nuestra, diversa y plural, los diseños curriculares oficiales tienen que ser forzosamente abiertos, de ahí el planteamiento de niveles de concreción curricular, que a medida que se aproximan a la realidad de las aulas dependen más de contextos específicos, de los centros $\mathrm{y}$, finalmente, de las decisiones de los profesores.

Consideramos que el profesor es un fuerte determinante desde la configuración del proyecto Curricular de Centro y la elaboración de las programaciones hasta su puesta en práctica en las aulas, y que en todos estos momentos el profesor toma decisiones de acuerdo con sus valores.

Nuestro propósito es conocer los valores del profesorado al término de su formación inicial y los que estima que debe fomentar entre sus alumnos, de donde surge la necesidad de elaborar instrumentos que permitan saber cuáles son los valores del profesorado y en qué grado los posee, así como cuáles y en qué grado son los valores que desea transmitir a sus alumnos. Dada la reconocida necesidad de educación integral, así como la relación entre valores y educación, tales instrumentos 
de recogida de datos deben tratar de abarcar distintas categorias de valores vinculadas con las diferentes dimensiones de la persona.

Por todas las razones aludidas, nos proponemos dar respuesta a dos bloques de objetivos correspondientes a un mismo objeto de estudio que tratamos desde una doble vertiente, teórica y empírica.

En el marco teórico, de donde se extrae la necesaria fundamentación para la parte empírica, tratamos aspectos conceptuales sobre axiología y sobre axiología educativa, consideramos las interrelaciones y posibles respuestas educativas ante los valores del contexto social, analizamos el concepto y los valores propios de un modelo de educación integral $\mathrm{y}$, finalmente, se presenta la figura del profesor como agente transmisor de valores en el sistema educativo formal.

La segunda parte de nuestro trabajo sigue un criterio empírico. En ella tratamos de constatar los valores de un grupo, tal y como se presentan en la realidad. El ámbito de aplicación es el alumnado del último curso de Magisterio de todas las especialidades de la Facultad de Ciencias de la Educación de la Universidad de Granada y el alumnado de último curso de las Facultades de Ciencias y Filosofía y Letras, de acuerdo con las titulaciones y especialidades de las distintas facultades más vinculadas tradicionalmente con la docencia.

Hemos seguido una metodología por encuesta utilizando dos cuestionarios de valores elaborados por nosotros y que reúnen los requisitos técnicos para poder ser utilizados: sistema de expertos y análisis factorial para el estudio de validez. Los instrumentos de recogida de datos se construyen de acuerdo con las características de los valores (polaridad, categorización, jerarquía, contenido, gradación) y siguiendo un modelo de educación integral cuyo contenido está tomado tanto de la realidad como de la literatura sobre valores y sobre axiología educativa. El primero de ellos se refiere a los valores que los encuestados han adquirido mediante su educación ("Valores adquiridos en mi educación"), el segundo a los valores que fomentarian como profesores ("El comportamiento de la persona educada"). Entre ambos cuestionarios existe un paralelismo en cuanto al contenido de los ítems de cada uno de ellos, de modo que sea posible la comparación.

Las puntuaciones otorgadas en uno y otro cuestionario se estudiaron en primer lugar para toda la muestra y en segundo lugar agrupados por variables: facultad, especialidad, sexo e intención de dedicarse a la enseñanza.

De acuerdo con estas variables constatamos:

$1^{\circ}$ La jerarquía de valores que manifiestan haber adquirido los alumnos de nuestra muestra de análisis de datos.

$2^{\circ}$ La jerarquía de valores que transmitirían mediante la educación.

3ㅇ Una vez establecidas ambas jerarquías, se procede al estudio de diferencias entre los valores expresados en uno y otro cuestionario.

$4^{\mathrm{o}}$ Finalmente se estudia la influencia de la variable especialidad y las posibles diferencias según las variables facultad, especialidad, sexo e intención de dedicarse a la enseñanza (variable "docencia").

Para establecer las jerarquías de valor estudiamos las puntuaciones medias de cada categoría. 
Cuando se trata de contrastar diferencias entre las medias de ambos cuestionarios, ya sea para el total de la población, ya para sus diferentes estratos, se utiliza la prueba t de Student, como en el caso de las diferencias por sexo. El análisis de varianza se utiliza cuando se trata de constatar si hay diferencias entre facultades, entre especialidades de una misma facultad, o entre alumnos de magisterio con los que manifiestan percibir como salida profesional la docencia y los que prefieren otras salidas profesionales.

Para realizar el estudio estadístico hemos recurrido al soporte informático (SPSS).

\section{CONCLUSIONES}

Las conclusiones de la parte teórica consisten en la exposición a modo de síntesis de las ideas más relevantes que se desprenden del estudio realizado. En cuanto a la parte empírica, que es nuestra aportación original, destacaremos los resultados siguientes:

Los valores que posee el total de la muestra estudiada coinciden con los de nuestro modelo, a los que se añaden el aprecio por la familia y la convivencia pacífica y respetuosa entre las personas, independientemente de sexo, raza, nacionalidad, creencias e ideologias.

Todas las categorías presentadas se consideran valiosas, pero no en la misma medida, sino jerarquizadas en el orden siguiente: 1. Ecológicos. 2. Morales. 3. Económicos. 4. Sociales. 5. Afectivos. 6. Vitales. 7. Estéticos. 8. Individuales. 9. Trascendentes. 10. Comunitarios. 11. Instrumentales. 12. Intelectuales.

Los valores ecológicos fueron siempre, salvo contadas excepciones, en ambos cuestionarios y para todas las variables contempladas, los que reci-

(C) Ediciones Universidad de Salamanca bieron las puntuaciones más altas, lo que viene a reflejar la atención de este grupo sobre un foco problemático de nuestra realidad actual.

En general, y aunque las diferencias no fueron siempre significativas, se aprecia una tendencia bastante frecuente para todas las variables a situar los valores personales (la realidad), por encima de los valores de la persona educada (el ideal). Esta tendencia es más fuerte para Magisterio, para la variable "mujer" y para Filosofía y Letras.

En cuanto a las variables estudiadas, hay que destacar los siguientes hallazgos:

- La especialidad resultó, en general, poco diferenciadora cuando se trata de una misma facultad.

- La carrera elegida determina en gran medida los valores, resultando éstos en ambos cuestionarios siempre más altos para Magisterio, con algunas excepciones, y Filosofía y Letras, lo que reveló mayor afinidad en la percepción de los valores entre las carreras más vinculadas a las letras.

- Las diferencias entre las tres facultades fueron menores cuando se trata de los valores personales que cuando se trata del modelo de persona educada.

- Magisterio y Filosofía marcaron fuertes diferencias respecto a las licenciaturas de Ciencias cuando se trata de los valores que se asignan a la persona educada. Las medias de Magisterio resultaron siempre superiores, como cabía esperar de la especialización propia de estas diplomaturas.

- La variable sexo es altamente diferenciadora tanto de los valores personales como de los asignados al modelo de persona educada. Salvo la ex- 
cepción de los valores intelectuales adquiridos, en que no hay diferencias significativas, las medias de las mujeres superaron las de los hombres en todas las categorías de ambos cuestionarios.

- La variable "docencia" mostró semejanza entre las licenciaturas para ambos cuestionarios, incluso en las jerarquías, pero resultó diferenciadora de las diplomaturas respecto a las licenciaturas. La comparación del modelo de persona educada entre Magisterio sólo con los alumnos de las licenciaturas que perciben la enseñanza como salida profesional mostró las jerarquías muy semejantes, pero diferencias significativas entre las medias de las categorías, es decir, un modelo de persona educada muy similar, pero mayor exigencia por parte de los maestros.

Las puntuaciones medias obtenidas con los instrumentos de medida elaborados expresaron siempre acuerdo o fueron equivalentes al acuerdo. En consecuencia, la parte empírica de nuestro trabajo pone de relieve la vigencia, entre nuestra muestra, de las categorías de valor presentadas. Ello, unido al bajo porcentaje de anotaciones en el espacio reservado para respuestas libres, nos da idea de la representatividad de estos valores respecto a la situación axiológica presente, al mis-mo tiempo que pone de relieve una gran coherencia entre los valores personales y los valores asociados al perfil de la persona educada.

La coherencia entre los propios valores y los que se tiende a fomentar mediante la educación, aunque las jerarquías no necesariamente coinciden, es indicativa de que conocer los valores personales del profesorado permitirá anticipar los que tratará de transmitir a los alumnos.
El modelo en que basamos nuestros cuestionarios es una propuesta de educación integral, de ahí que, siendo siempre expresiones de acuerdo las puntuaciones medias que reciben las categorías, podamos afirmar que los valores que se asocian a la persona educada cubren una gama suficientemente amplia para abordar las diferentes dimensiones de la personalidad humana. Por tanto, nuestra muestra de análisis de datos tendería a fomentar en sus alumnos un modelo de educación integral, condición, como dijimos, para una educación de calidad.

JIMÉNEZ RODRÍGUEZ, Miguel Ángel: $E l$ desarrollo personal. Un programa educativo aplicado en talleres de integración socio-laboral. Departamento de Teoría de la Educación. Universidad de Valencia. Septiembre de 1998. (Tesis Doctoral dirigida por el Dr. D. Bernardo Gargallo López, presidida por el Dr. D. Juan Escámez Sánchez.)

Este trabajo de investigación se sitúa ante la necesidad de responder a la carencia más importante de los grupos humanos que integran la población de los Talleres de Integración Socio-Laboral (TIS), que es la formación de la propia persona, y a hacerlo desde la Pedagogía. Dichos talleres se dirigen principalmente a poblacionés de jóvenes de barrios de acción preferente y persiguen la capacitación en diversos oficios, ofreciendo al tiempo una beca por día asistido, lo que los convierte en una opción atractiva para los jóvenes.

El autor diseñó un programa que se puso en práctica desde principios de abril a finales de noviembre de 1996, y el marco pedagógico en el que se desa- 
rrolló posibilitó una organización general de los talleres que facilitó la inclusión de actividades formativas (como el propio Programa de Desarrollo Perscnal) al mismo nivel de importancia curricular que el oficio impartido en el taller. Esta iniciativa, junto con el trabajo en equipo de los educadores, orientados y dirigidos por el autor, ha posibilitado su implementación con garantías de éxito.

La investigación se aborda desde la pretensión de constituirse en una propuesta pedagógica global que cubra los aspectos fundamentales que pueden dotar a la persona de eficacia ante la vida. Éstos son el trabajo sobre sí mismo (autoconcepto, autoestima, autorrealización, capacidad para la toma de decisiones), la relación con los demás (comunicación, asertividad, amistad, amor, sexualidad) y la actuación ante las distintas situaciones de la vida (trabajo-ocio, salud, familia, opciones de vida, aprender a consumir, participación social).

El punto de partida es, además del análisis de la realidad para volver a ella transformándola, el tratar de llevar adelante la intención última de los talleres, que es la integración.

La base teórica de la tesis se sustenta, desde la filosofia, en el personalismo mounieriano y en su énfasis puesto en la importancia central de la persona, capaz de ir progresando dialécticamente en un continuo que se dilata hasta la dimensión trascendente de la existencia humana. Desde la psicología lo hace en la corriente sociopersonal. A nivel pedagógico el autor opta por una posición ecléctica al aplicar principios de diversas teorías que pueden ser utilizados para construir un programa que responda eficazmente a las características estructurales de los talleres, de su población y a los objetivos formativos que se pretenden alcanzar.

El diseño de la investigación es cuasi-experimental, con tres talleres que constituyen el grupo de control y otros tres el experimental. Se aplicaron diversas pruebas que barrieron complementariamente los diversos aspectos tratados en el programa y se llevaron a cabo comparaciones y análisis intra e intergrupo procediéndose también a una comparación homóloga por pares de talleres de características similares, en el pretest, en el postest y en pretestpostest.

Los resultados no muestran muchas diferencias significativas pero sí presentan una clara tendencia a favor del grupo experimental.

El trabajo abre una línea de intervención e investigación en el campo de la Pedagogía Social donde no abundan los materiales sistemáticamente estructurados y validados empíricamente, y ofrece una herramienta de trabajo sólida en manos de los educadores que pretendan formar personas pertenecientes a ambientes sociales deprimidos -aunque también ha sido aplicado con éxito posteriormente en poblaciones más normalizadas-, donde esta dimensión cobra especial interés. Lo mejor que se puede decir del programa es que ha permitido, en diferentes talleres, afrontar con éxito la labor de formación humana integral de un modo específico y cercano a los intereses de los destinatarios.

MORALES GÓMEZ, A. (1998): Sistematización de las principales tecnologias de intervención pedagógica. Departamento de Pedagogía. Univer- 
sidad de Granada. Diciembre de 1997. (Tesis Doctoral dirigida por el Dr. D. Salvador Peiró i Gregori; presidida por el Dr. D. Ricardo Marín Ibáñez.)

El presente trabajo de investigación trata de informar sobre las principales tecnologías de intervención pedagógica a fin de facilitar al profesorado la elección de la tecnología más adecuada.

El autor ha realizado un estudio funcional de una de las principales tecnologías de aplicación al entorno social (Problem Solving), base del Programa de Intervención, cuyo objetivo era mejorar los problemas de adaptación y prevenir el fracaso escolar.

El planteamiento consistía en educar a los hijos mediante la reeducación de los padres, a fin de potenciar las capacidades educativas de la familia.

La Tesis se divide en tres partes: la primera se dedica a las bases de partida de la investigación; la segunda presenta los supuestos básicos de la intervención educativa, junto con un análisis de las principales tecnologías de intervención pedagógica, y la tercera parte se centra en la elaboración, aplicación y evaluación de un programa de intervención en el aula.

El primer capítulo se dedica a analizar los procesos educacionales.

En el segundo capítulo se establece el marco teórico de intervención en la acción educativa y se realiza un análisis de las principales tecnologías de intervención pedagógica.

En el capítulo tercero se efectúa una sistematización de dichas tecnologías, con un apartado dedicado al uso que han hecho de las mismas diversos autores.

(C) Ediciones Universidad de Salamanca
Todo el capítulo cuarto se reserva a un estudio funcional del problem-solving, a partir de las investigaciones y experiencias que existen sobre educación de padres y resolución de problemas de conducta.

El capítulo quinto está dedicado al programa de intervención en el aula. Se describe la metodología de investigación y los instrumentos empleados en la evaluación del trabajo: TAMAI (Test autoevaluativo multifactorial de adaptación infantil), CAEP (Cuestionario adaptado del estilo educador parental), ESDA (Entrevista semiestructurada de diagnóstico de la adaptación) y CAC (Cuestionario de autoevaluación del cambio).

Hay un estudio previo de cada alumno, seguido de las acciones y programas de intervención utilizados.

En la recogida de datos, los resultados obtenidos confirman la hipótesis de trabajo: La aplicación del Problem Solving, a través del estudio de casos y el método "nadie pierde", contribuye significativamente a la resolución de problemas de adaptación y sirve para prevenir el fracaso escolar, a un nivel del 0,05 .

En el capítulo sexto se exponen las conclusiones de la investigación realizada.

En el apartado reservado para Anexos, destaca un recurso pedagógico novedoso, que el autor denomina TebeoPedagógico: libro-resumen, ilustrado, escrito y encuadernado por el propio alumno.

PELUFO ENGUIX, I. Cristina: Mejora de los bábitos de estudio en universitarios de primer curso en un Colegio Mayor. Departamento de Teoría de 
la Educación. Universidad de Valencia. Octubre de 1996. (Tesis dirigida por el Dr. D. Bernardo Martínez Mut, presidida por el Dr. D. Juan Escámez Sánchez.)

En el sistema educativo español la tutoría forma parte de la función docente. Pero en la enseñanza universitaria, al contrario de lo que ocurre en la educación infantil, primaria y secundaria, no encontramos un marco legal que dé soporte al régimen de tutorias; únicamente se menciona la tutoría como un servicio que el docente debe prestar a sus alumnos y que se especifica de modo más concreto en la normativa académica de cada una de las universidades, donde, en general, se hace referencia al número de horas que se dedican a esta tarea.

Pensamos que la tutoría es un medio educativo que debe ser desarrollado y utilizado en la enseñanza superior. En el ámbito universitario, detectamos que los alumnos de primer curso tienen necesidades personales, académicas y vocacionales que pueden ser impulsadas desde la acción tutorial. Y creemos que los Colegios Mayores Universitarios, que en su papel educativo se han distinguido por su carácter de complementariedad con la universidad, son un ámbito de educación no formal idóneo para llevar a cabo esta función de acción tutorial.

El objetivo principal de este estudio es evaluar la eficacia de un programa de intervención educativa para la mejora de los hábitos de estudio en los universitarios de primer curso, a través de la tutoría desarrollada en un Colegio Mayor.

El trabajo consta de dos partes: en la primera, se propone el marco teóri- co-conceptual que sirve de justificación a la investigación. En ella se analizan los referentes teóricos que incluyen una descripción de la organización y funciones de los Colegios Mayores, el análisis de los factores que intervienen en el aprendizaje, hábitos de estudio, la acción tutorial y sus estrategias metodológicas. En la segunda, aparece el diseño de la investigación, se acota el objeto de estudio y se describen las distintas fases del programa de intervención.

La tesis se estructura en ocho capítulos. En los tres primeros se describe el marco teórico-conceptual de la investigación. El capítulo cuarto relata: ción,

a) el diseño del plan de investiga-

b) el ámbito de la investigación que son los universitarios de primer curso, residentes en un Colegio Mayor, a los que elegimos deliberadamente por las necesidades específicas que plantean cuando ingresan en la universidad. Seleccionamos los grupos de nuestra investigación, que son, por un lado, el grupo programa, que es un grupo natural ya constituido: los residentes de primer curso del Colegio Mayor La Asunción de Nuestra Señora de Valencia, y de otro lado, como grupo control se selecciona un grupo homogéneo al grupo programa,

c) los instrumentos de medida: Cuestionario de Hábitos de Estudio ACH-73, del Test de Aptitudes Diferenciales del Razonamiento Abstracto y el Razonamiento Verbal.

d) se enuncian las hipótesis de trabajo,

e) las metas a conseguir, y

f) se describen los criterios de evaluación. 
El capítulo quinto se dedica al análisis de la realidad previa a la ejecución del programa.

En el capítulo sexto se describe la planificación y ejecución del programa. La metodología utilizada se basa en la motivación, reflexión y autocontrol, a fin de fomentar actitudes y condiciones personales positivas de los alumnos que les faciliten la realización de su proyecto personal y académico, así como la adquisición e incorporación a su tarea diaria de aquellas técnicas y hábitos que hagan su estudio más eficaz. Por otro lado, se ofrece soporte, formación e información técnico-pedagógica a los tutores que llevan a cabo el programa de intervención.

El programa consta de ocho sesiones de trabajo con los tutores, destinadas a formarlos en las estrategias metodológicas de la acción tutorial y trabajo en equipo, así como en las técnicas y conocimientos necesarios para dar soporte al desarrollo del programa. Cada sesión de trabajo viene operativizada en forma de fichas donde aparece: el objetivo, las actividades y el material a utilizar.

Con los alumnos se realizan ocho sesiones grupales, que vienen operativizadas de igual modo que las de los tutores, y doce individuales en las que se lleva el seguimiento de las metas fijadas con cada alumno para la mejora de sus hábitos de estudio. Estas sesiones se apoyan en un soporte documental confeccionado expresamente para el programa y que facilita la unificación de criterios de actuación de todos los tutores. Se desarrollan con una periodicidad quincenal.

El capítulo séptimo, detalla los resultados obtenidos tras la ejecución del programa, que confirman las hipótesis planteadas, encontrando que los alumnos que han seguido el programa: a) mejoran sus actividades frente a la clase y el estudio, b) mejoran las condiciones que favorecen la propia situación del estudio a través de un mayor autocontrol, c) incorporan técnicas de estudio eficaces en cuanto a la lectura y recogida de datos y en los recursos bibliográficos. Al mismo tiempo, se ha conseguido una mejoría significativa en sus capacidades intelectuales, concretamente en el razonamiento abstracto.

TOUZA RODRÍGUEZ, María Inés: Los inmigrantes y su inserción laboral en la Comunidad Autónoma de Madrid: propuesta de un proyecto pedagógico. Departamento de Teoría e Historia de la Educación. Universidad Complutense. Enero de 1998. (Tesis doctoral dirigida por el Dr. D. José Ángel López Herrerías.)

La llegada de inmigrantes a nuestro país y a la Comunidad Autónoma de Madrid, en particular, nos obliga a ofrecer soluciones que les faciliten una inserción eficaz en el Mundo Laboral, partiendo de la elaboración de programas formativos concretos. Éste es el objetivo último de esta investigación que se centra en los INMIGRANTES SIN ESTUDIOS O CON ESTUDIOS PRIMARIOS que son los que tienen mayores dificultades a la hora de acceder a un puesto de trabajo por carecer de una Formación Ocupacional apropiada.

A este fin se realizó un análisis de las ocupaciones con mayor índice de colocación en nuestra Comunidad y en las que podemos, previa formación, ubicar a nuestro colectivo de inmigrantes. Se tomó como punto de partida el Observatorio de Ocupaciones del INEM 
y se extrajeron las diez ocupaciones con mayor índice de colocación.

Este análisis fue indispensable para la elaboración y el desarrollo de Programas de Formación dirigidos específicamente a nuestro colectivo puesto que concebimos la Formación como un Programa de Empleo, es decir, como un instrumento que facilite la inserción profesional. Consideramos que la planificación de la Formación Ocupacional ha de basarse en indicadores activos del Mercado de Trabajo: análisis de ofertas de empleo no cubiertas, nuevas ocupaciones, etc.

En el caso de nuestro colectivo inmigrante $\mathrm{y}$, teniendo en cuenta que su punto de partida para el pleno acceso a la integración está comprometido por una serie de déficits entre los que prima el desconocimiento de la lengua y cultura de la sociedad de acogida, abogamos por una Formación de Base destinada a superar las deficiencias del Sistema Educativo Básico. Esta Formación de Base ha de tener una proyección poliva- lente que le permita el acceso a puestos de trabajo, muchas veces alejados de su profesión original, y que esté abierta a un Mercado Laboral cambiante. Sin olvidar un respeto a la individualidad y una flexibilidad que se reflejará, tanto en el diseño curricular como en los recursos humanos y materiales.

Conscientes de la situación de nuestro colectivo, elaboramos un Proyecto Pedagógico que, además de permitir el acceso al empleo, cubre una serie de necesidades que ayudan a adaptarse a la evolución del Mercado de Trabajo y a sentirse personas involucradas en la sociedad receptora. Elaboramos también una Guía Didáctica como material impreso de apoyo.

La metodología de nuestra investigación tiene un carácter exploratorio que, apoyándose en una documentación previamente analizada, criticada y valorada, da pie a la elaboración del Proyecto Pedagógico que busca la inserción laboral del colectivo inmigrante. 